\title{
PLEIONE AS A VARIABLE STAR
}

\author{
A. S. SHAROV and V. M. LYUTY \\ Moscow University, Moscow, U.S.S.R.
}

The well-known shell star Pleione is also an interesting variable star, BU Tau. Its variability was first discovered by Calder (1937) from photoelectric observations in the 1930's. The light curve of this star from the end of the 19th century to 1954 was studied by Binnendijk $(1949,1955)$. Botsula and Sharov (1959) and Sharov (1961) added some old and new observations and continued the light curve up to 1959.

Variations of Pleione are connected with the absorption of the starlight in the shell material. Possibly this phenomenon also took place in 1883-1884, when the star was faint according to the observations of Lindemann (1884). Near that time hydrogen emission lines were observed in the spectrum of the star. Between the two decreases the brightness of the star was practically constant. The well observed ejection of the shell in 1936 led to the decrease of light during the period from 1937 to 1958 . Some observations of Pleione were published after 1958. In autumn 1971 we started systematic photoelectric $U B V$ observations of Pleione at the Crimean station of the Sternberg Astronomical Institute (Sharov and Lyuty, 1972a, 1972b, 1973, 1975). It is interesting to note that the decrease of Pleione was found during the second night of observations! Now these observations are continuing. The light curve from all our observations up to April 1975 is presented in Figure 1. The decrease in brightness lasted for two years, and the minimum took place at the end of 1973. The brightness

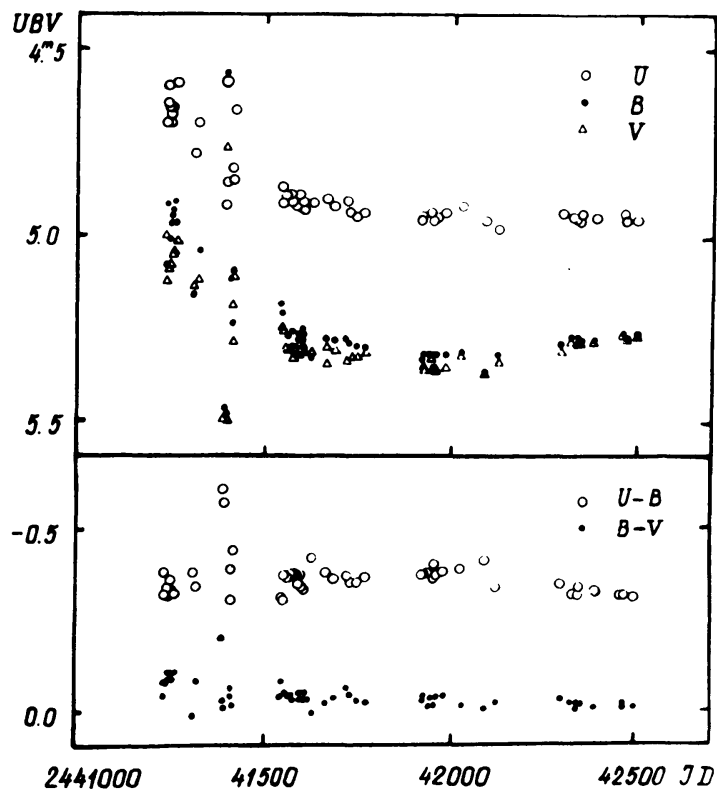

Fig. 1. Light curve of Pleione from photoelectric observations from 1971 to 1975. 
in $B$ and $V$ then started to increase slowly, but in $U$ the star is still at minimum. We have an opportunity to observe a new shell phase of Pleione photometrically from the beginning in 1971. The appearance of the absorption shell spectrum of Pleione was observed a year later, in December 1972 (Morgan et al., 1973), and thereafter.

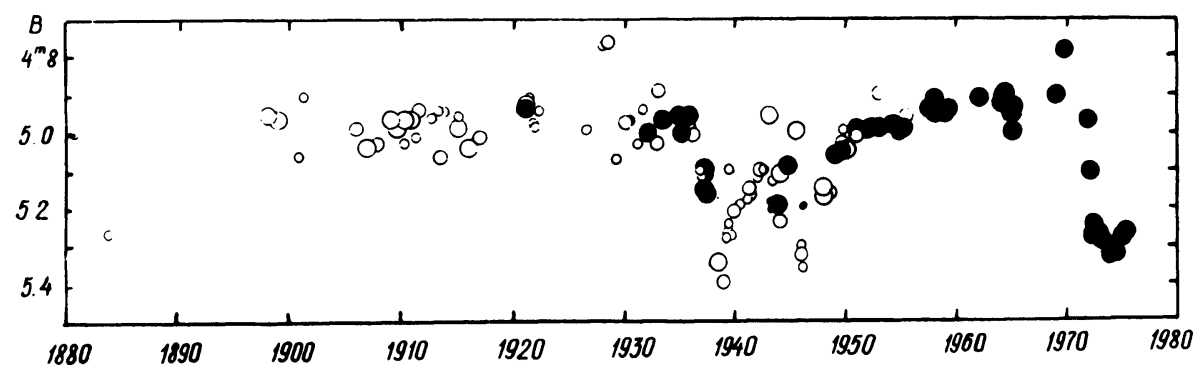

Fig. 2. Total light curve of Pleione from 1880 to 1975 . Filled circles represent photoelectric observations.

The total light curve of the star is presented in Figure 2. We used all the published material (Botsula and Sharov, 1959; Sharov, 1961; Sharov and Lyuty, 1972b, 1973, 1975; Haupt and Schroll, 1974; Johnson et al., 1966). Here we give average magnitudes from our observations. The observations of Johnson et al. need some systematic corrections.

During a period of less than one century, astronomers have observed two (or three) shell outbursts of Pleione. The previous case was studied in detail. We hope also that new photometric and spectral observations will give much interesting information about the nature of Pleione.

\section{References}

Binnendijk, L.: 1949, Astron. J. 54, 117.

Binnendijk, L.: 1955, Astron. J. 60, 364.

Botsula, R. A. and Sharov, A. S.: 1959, Perem. Zvezdy 12, 398.

Calder, W. A.: 1937, Ann. Harv. Coll. Obs. 105, 453.

Haupt, H. F. and Schroll, A.: 1974, Astron. Astrophys. Suppl. Ser. 15, 311.

Johnson, H. L., Mitchell, R. I., Iriarte, B., and Wisniewski, W. K.: 1966, Commun. Lunar Planet. Lab. 4, No. 63, 99.

Lindemann, E.: 1884, Mém. Acad. Imp. Sci. St-Petersb., ser. VII, 32, No. 6.

Morgan, W. W., White, R. A., and Tapscatt, J. W.: 1973, Astron. J. 78, 302.

Sharov, A. S.: 1961, Perem. Zvezdy 13, 443.

Sharov, A. S. and Lyuty, V. M.: 1972a, Inform. Bull. Var. Stars, No. 698.

Sharov, A. S. and Lyuty, V. M.: 1972b, Perem. Zvezdy 18, 377.

Sharov, A. S. and Lyuty, V. M.: 1973, Inform. Bull. Var. Stars, No. 814.

Sharov, A. S. and Lyuty, V. M.: 1975, Astron. Circ., No. 872. 